spp., P timonensis and Eggerthella spp., were strongly associated with all four clinical signs defined by Amsel's criteria.

Conclusion Lactobacillus species including $\mathrm{L}$ iners and $\mathrm{L}$ gasseri are the predominant vaginal species found in the vaginal tract of Indian women of reproductive age. A heterogeneous vaginal community marked by the presence of $G$ vaginalis, Sneathia spp., Atopobium spp., Prevotella spp., Eggerthella spp., is associated with BV and its clinical symptoms.

\section{P364 ACCESS TO HIV-PREVENTION IN FEMALE SEX WORKERS IN UKRAINE BETWEEN 2009 AND 2017: COVERAGE, BARRIERS AND FACILITATORS}

N Blumer*, J Burns, L Pfadenhauer. Ludwig-maximilians-Universität Munich, Munich, Germany

10.1136/sextrans-2021-sti.406

Background The comprehensive prevention package (PP) is an important intervention in reducing the burden of HIV amongst female sex workers (FSW) worldwide. According to the UNAIDS 90-90-90 goal, 90\% of people at risk of HIV infection were to have access to the PP by 2020, however, in 2015 and 2017, only 46\% and 48\% of Ukrainian FSWs accessed the PP. Hence, we aimed to identify the barriers and facilitators associated with the access to HIV prevention amongst Ukrainian FSWs between 2009 and 2017.

Methods We began by conducting a comprehensive literature review to develop a literature-informed conceptual framework. We then conducted a document analysis to identify the items of the Ukrainian prevention package (PP). Identified by our conceptual framework, we then conducted descriptive analyses using the Integrated Bio Behavioural Surveillance Surveys, to explore PP coverage from 2009 to 2017 and the influence of factors associated with access to the PP.

Results Our conceptual framework illustrates that most thematic clusters which influence access to HIV prevention operate at the macrostructural level. 24 prevention items were included in the PP in 2012 and 2013, however this dropped to 7 items in 2014. Access to the Ukrainian PP increased from 2009 to 2013, where it peaked at 67.42\%, however dropped to $32.10 \%$ in 2017 . Our analyses of the IBBS identified that being a client of a non-governmental organisation, street and highway solicitation, non-condom use, and knowledge of HIV were associated with access to HIV prevention in the Ukrainian context.

Conclusions Our novel study calls for colleagues designing future HIV prevention interventions to strongly consider the barriers and facilitators identified in our conceptual framework and the multiple structural levels on which barriers and facilitators operate. Colleagues can draw upon our findings to bolster the level of access for FSWs in their own countries.

\section{P365 GENDER AND SEX - WHAT SHOULD BE MEASURED AND HOW? LEARNINGS FROM BRITAIN'S NATIONAL SURVEY OF SEXUAL ATTITUDES AND LIFESTYLES}

${ }^{1,2} \mathrm{~S}$ Clifton, ${ }^{3} \mathrm{~K}$ Nambiar, ${ }^{2} \mathrm{M}$ Karikoski, ${ }^{1} \mathrm{~L}$ McDonagh, ${ }^{1} \mathrm{~J}$ Gibbs ${ }^{*},{ }^{2} \mathrm{C}$ Lapham, ${ }^{1} \mathrm{P}$ Sonnenberg, ${ }^{1} \mathrm{C}$ Mercer. 'University College London, London, UK; ${ }^{2}$ Natcen Social Research, London, UK; ${ }^{3}$ Brighton and Sussex University Hospitals NHS Trust, Brighton, UK

10.1136/sextrans-2021-sti.407
Background Many social surveys have purported to measure 'sex', but this has been through interviewers' observation of outward appearance (e.g. clothing, name). In previous rounds of the decennial large-scale probability sample surveys of the British general population (www.natsal.ac.uk), this approach was used to make assumptions about the respondents' anatomy in the rest of the questionnaire. This is not appropriate and results in some misclassification. Although often used interchangeably, sex and gender refer to different concepts, and can differ. Here, we consider what aspects of sex and gender are relevant for a sexual health survey, and how these can be measured in a way that captures diversity, is acceptable, and is comprehensible to the general population. This informed development of questions for Natsal-4.

Methods First, we reviewed international literature for questions about gender/sex in social surveys, censuses and sexual health research, including national (BASHH) recommendations. Second, we conducted stakeholder engagement. Third, we synthesised the findings from both activities into questions deemed appropriate for use with general population samples. Finally, questions were cognitively tested among 30 members of the general population (including three people who identified as trans/had a trans history).

Results Several questions from the literature had undergone general population testing, however, there were no definitive or 'harmonised' questions. No existing set of questions captured the elements of sex and gender most relevant for a sexual health survey. Few studies addressed how to tailor detailed sexual partnership questions to account for trans partners. Our developed questions were found to be well understood and accepted by both trans and non-trans people in cognitive testing. Discussion We used a multi-stage process to identify a set of questions that seem to balance the needs of a diverse group of participants. These questions are subject to further piloting before being finalised for inclusion in Natsal-4.

\section{P368 ETIOLOGY OF CERVICITIS - ARE THERE NEW AGENTS IN PLAY?}

A Bhargava*, N Khunger, S Bansal, P Panchal, N Joshi. VM Medical College and Safdarjung Hospital, New Delhi, India

\subsection{6/sextrans-2021-sti.408}

Background Considering the changing causative and resistance pattern of agents implicated in Sexually Transmitted Infections (STIs), etiological diagnosis is imperative especially in countries practicing syndromic management. This study was designed to diagnose cervicitis and to identify etiological agents associated with cervicitis.

Methods Female STI clinic attendees presenting with cervicovaginal discharge were examined for presence of cervicitis as per standard protocol. Endocervical swabs were collected for gram staining and Real time PCR was performed for various bacterial and viral STI agents in patients presenting with cervical discharge and clinical signs of cervicitis. A vaginal swab was also evaluated for bacterial vaginosis by Nugent's criteria. Results Of 64 patients with clinical cervicitis, $26.6 \%$ and $12.5 \%$ patients complained of genital itching and lower abdominal pain respectively. Mean of 36.6 pus cells/hpf were observed, appreciably greater number in patients with N.gonorrhoeae (NG) and C.trachomatis infections ( $\mathrm{p}$ value 0.0063 and 0.0032 respectively). Pus cells were high (mean 68 pus 
cells/hpf) in patients with U.urealyticum, though this may be attributed to coexisting NG. Agents isolated from endocervix were Ureaplasma parvum 26(40.6\%), N.gonorrhoeae, 17 (26.6\%), Mycoplasma hominis 11(17.2\%), HSV2, 9(14.1\%), Ureaplasma urealyticum 5(7.8\%), T.vaginalis 4(6.3\%), HSV1 and C.trachomatis, 1 each(1.6\%) and Mycoplasma genitalium $(0 \%)$. Bacterial vaginosis was diagnosed in $14(21.9 \%)$ patients. Multiple, two, three, four and five agents were isolated in 10 , 6, 6 and 1patients respectively. Isolation of M.hominis and U. parvum was significantly associated with bacterial vaginosis ( $p$ value $0.04 \& 0.003$ respectively). Non-usage of condoms predisposed to cervicitis.

Conclusion Few C.trachomatis isolates, absence of M.genitalium and increasing prevalence of HSV, highlights the changing etiological pattern of cervicitis. Though U.parvum and M. hominis are usually commensals their high isolation, association with bacterial vaginosis and propensity to cause diseases in preterm infants and extragenital infections puts forth the need for further studies and regular monitoring of agents implicated in cervicitis.

\section{P370 WHAT ARE THE RISK FACTORS FOR CHLAMYDIA IN WOMEN ATTENDING AN ABORTION SERVICE IN THE UK?}

${ }^{1,2} \mathrm{~F}$ Von Hawrylak*, ${ }^{1} \mathrm{R}$ Johnson, ${ }^{1} \mathrm{~A}$ Liebow, ${ }^{3} \mathrm{P}$ Muir, ${ }^{1} \mathrm{M}$ Singh, ${ }^{1,2} \mathrm{P}$ Horner. ${ }^{1}$ University Hospitals Bristol, Bristol, UK; ${ }^{2}$ Bristol University, Bristol, UK; ${ }^{3}$ Public Health England South West laboratory, Bristol, UK

10.1136/sextrans-2021-sti.409

Background The 2019 updated NICE abortion guidance suggested that prophylactic antibiotics should no longer be routinely given for medical abortions and instead recommended individual risk assessment.

Untreated Chlamydia trachomatis is an important cause of severe infection post abortion. In 2002, 75 (7.5\%) of 998 women having an abortion tested chlamydia-positive using a nucleic acid amplification test (NAAT). Women attending our abortion service have been tested for Chlamydia using a NAAT. We wished to obtain contemporary estimates of Chlamydia prevalence and examine associated risk factors.

Methods Data from 13,427 women aged 15-50 years having an abortion between April 2010 and March 2020, was retrospectively analysed (SPSS, IBM). Ethics approval was sought and all data was anonymised.

Results Of 13,337 with Chlamydia NAAT results available, $4.2 \%$ (565) were Chlamydia-positive. Chlamydia was associated with younger age $(\mathrm{p}<0.0001$, Chi-square $)$ thus $7.1 \%$ $(164 / 2135)$ of $15-19$ year olds, 5.5\% (222/3782) of 20-24 year olds and $2.5 \%(174 / 6774)$ of women over 24 years old were Chlamydia-positive. Chlamydia detection was associated with 2 or more partners within the past year in women aged 20-24 years old, $8 \%(94 / 1181)$ vs $4.6 \%$ (106/2304) $(\mathrm{p}<0.0001$, OR 1.8; 95\%CI, 1.35-2.39) and women over 25 years old, $4.2 \% \quad(53 / 1270)$ vs $2.1 \% \quad(95 / 4507)(\mathrm{p}<0.0001$, OR 2.0 ; 95\% CI 1.44-2.85) but not women aged 15-19 years old, $7.8 \%(50 / 643)$ vs $6.1 \%(83 / 1367)(\mathrm{p}=0.27$ OR 1.3 ; $95 \%$ CI $0.91-1.9)$. For women who had a new partner in the last 3 months, findings were similar regardless of age.

Conclusion Chlamydia prevalence in women undergoing an abortion is lower than that observed in 2002 but is higher than the general population. Although Chlamydiawas associated with 2 or more partners in the previous year in women aged over 19 years, the association is weaker than that observed in the general population and no association was observed with partner change in women aged 15-19 years old

\section{P371 COMBINATION HIV/HCV/HBV/STIS PREVENTION AMONG MSM AND USE OF MOBILE APPLICATIONS/SOCIAL NETWORKS AT THE COVID-19 CONDITIONS IN UKRAINE}

A Chernyshev*, A Bogoslavets, R Marchenko. Alliance.Global, 62B, B.Kchmelnitskogo str., Ukraine

\subsection{6/sextrans-2021-sti.410}

Background/Purpose Restrictions of COVID-19 pandemic have made it more difficult to provide HIV testing and PrEP services to MSM in large Ukrainian cities. The purpose of the intervention is to maintain the effectiveness of the provision of these services during COVID-19 and to implement new/ innovative and more mobile interventions in this regard.

Approach The methodology consists of conducting two national campaigns aimed at MSM recruiting in 3 largest cities of Ukraine, for conducting of HIV/STI/HCV testing, as well as to attract to the PrEP program, through targeted advertising on gay dating applications and social networks for directing to web-resources https://gettest.com.ua and https://prep. com.ua, for passing of testing and/or to receive equal counseling on PrEP, at Alliance.Global's testing points. All confirmed HIV-positive MSM have been provided a social support to receive ART; for HIV-negative MSM, we proposed to become a member of the free PrEP program.

Outcomes/Results Thanks to advertising on two web resources, during September-December 2020, 841 MSM registered for HIV/HCV/STI testing through the GetTest website and $80 \%$ of them were tested (the number of HIV-positive results was approximately 4\%). 3244 MSM have learned about the PrEP program during this period, approximately 300 new MSM have been attracted to the PrEP program (the coverage of the PrEP intervention in 2020 amounted about 1,300 MSM).

Innovations/Conclusions Thanks to the introduction of two advertising campaigns on the Internet, at the COVID-19 conditions, as well as such innovative interventions as delivery of clients by taxi to receive PrEP in a medical institution, receiving the free premium accounts in the mobile gay application Hornet, compliance with sanitary norms (free masks, disinfectants, etc.) and mandatory pre-registration (to avoid queues), we were able to successfully saved services, and increase the intensity of testing and staging to PrEP for MSM compared to the first half of 2020 .

\section{P374 CHARACTERISTICS OF PREGNANT WOMEN, PARTNERS, AND PARTNER TREATMENT PREFERENCES AMONG WOMEN UNDERGOING SEXUALLY TRANSMITTED INFECTION SCREENING, RAWALPINDI, PAKISTAN}

${ }^{1}$ E Hansman* ${ }^{*},{ }^{1} \mathrm{~A}$ Chaudry, ${ }^{2} \mathrm{R}$ Chaudhri, ${ }^{2} \mathrm{~A}$ Kayani, ${ }^{1} \mathrm{~L}$ Hayes, ${ }^{3} \mathrm{C}$ Bristow, ${ }^{2} \mathrm{~K}$ Javaid ${ }^{2} \mathrm{~N}$ Khan, ${ }^{2} \mathrm{~S}$ Akhlaque, ${ }^{2} \mathrm{~B}$ Yasmeen, ${ }^{1} \mathrm{~J}$ Klausner. 'David Geffen School of Medicine, UCLA, Los Angeles, USA; ${ }^{2}$ Rawalpindi Medical University/Holy Family Hospital, Rawalpindi, Pakistan; ${ }^{3}$ University of California San Diego School of Medicine, San Diego, USA 